\title{
Semi-Minimal Invasive Method to Induce Myocardial Infarction in Rats and the Assessment of Cardiac Function by an Isolated Working Heart System
}

\author{
Patrick M. Pilz ${ }^{1}$, Miriam Lang ${ }^{1}$, Ouafa Hamza ${ }^{1}$, Petra L. Szabo ${ }^{1}$, Milat Inci ${ }^{1}$, Anne M. Kramer ${ }^{1}$, Michael Koch ${ }^{2}$, Johann $^{2}$ \\ Huber $^{3}$, Bruno K. Podesser ${ }^{1}$, Attila Kiss ${ }^{1}$ \\ ${ }^{1}$ Ludwig-Boltzmann-Institute for Cardiovascular Research at the Center for Biomedical Research, Medical University of Vienna ${ }^{2}$ Concept \& Ideation, \\ Electrical Sector/EMEA/Power Distribution Division, Eaton Industries (Austria) GmbH ${ }^{3}$ Vetfarm and Clinical Unit for Herd Health Management in Ruminants, \\ Clinic for Ruminants, Vetmeduni Vienna
}

\section{Corresponding Authors}

Bruno K. Podesser

bruno.podesser@meduniwien.ac.at

Attila Kiss

attila.kiss@meduniwien.ac.at

\section{Citation}

Pilz, P.M., Lang, M., Hamza, O., Szabo, P.L., Inci, M., Kramer, A.M., Koch, M., Huber, J., Podesser, B.K., Kiss, A. Semi-Minimal Invasive Method to Induce Myocardial Infarction in Rats and the Assessment of Cardiac Function by an Isolated Working Heart System. J. Vis. Exp. (160), e61033, doi:10.3791/61033 (2020).

\section{Date Published}

June 11, 2020

\section{DOI}

$10.3791 / 61033$

URL

jove.com/video/61033

\section{Abstract}

Myocardial infarction (MI) remains the main contributor to morbidity and mortality worldwide. Therefore, research on this topic is mandatory. An easily and highly reproducible $\mathrm{Ml}$ induction procedure is required to obtain further insight and better understanding of the underlying pathological changes. This procedure can also be used to evaluate the effects or potency of new and promising treatments (as drugs or interventions) in acute $\mathrm{MI}$, subsequent remodeling and heart failure (HF). After intubation and pre-operative preparation of the animal, an anesthetic protocol with isoflurane was performed, and the surgical procedure was conducted quickly. Using a minimally invasive approach, the left anterior descending artery (LAD) was located and occluded by a ligature. The occlusion can be performed acutely for subsequent reperfusion (ischemia/reperfusion injury). Alternatively, the vessel can be ligated permanently to investigate the development of chronic MI, remodeling or HF. Despite common pitfalls, the drop-out rates are minimal. Various treatments such as remote ischemic conditioning can be examined for their cardioprotective potential pre-, periand post-operatively. The post-operative recovery was quick as the anesthesia was precisely controlled and the duration of the operation was short. Post-operative analgesia was administered for three days. The minimally invasive procedure reduces the risk of infection and inflammation. Furthermore, it facilitates rapid recovery. The "working heart" measurements were performed ex vivo and enabled precise control of preload, afterload and flow. This procedure requires specific equipment and training for adequate performance. This manuscript provides a detailed step-by-step introduction for conducting these measurements. 
Although incidence is continuously decreasing, acute myocardial infarction (MI) is still the major contributor to morbidity and mortality worldwide ${ }^{1}$. There are restrictions on evaluating the efficacy of potential treatments as drugs or surgical procedures which prevent and treat acute MI. Before their effects can be examined in humans, these treatments must be tested for risks in advance, including in vivo examinations in animals. There is no better opportunity to study a pathology than under in vivo conditions. Therefore, $\mathrm{Ml}$ induction in rats or mice and even large animal models (pigs or sheep) permits the investigation of short- (acute) and long-term (chronic) changes due to ischemia in the coronary arteries and the surrounding myocardium, as well as systemic changes due to impaired cardiac function. Infarct size was previously the main objective, but more recently subsequent cardiac remodelling processes in acute $\mathrm{Ml}$ or ischemia/reperfusion injury as well as in consecutive heart failure (HF) have become of great interest. Therefore, a comparable and easily reproducible method is required to reach consistent outcomes.

While the use of cryo-ablation to gain MI has been reported ${ }^{2}$, our method builds on other studies in which investigators occlude the left anterior descending artery (LAD) by a single stitch ligation. In comparison to (hemi-)sternotomy procedures, the minimally invasive approach which will be presented in this article, permits a faster post-operative recovery and markedly reduces the operation time. A common step of other surgical procedures is the lift-out of the heart from the thorax to perform the heart stitch ${ }^{3}$. The approach of this method renders this step unnecessary. Depending on the protocol, two different procedures can be performed: a temporary occlusion using a tourniquet to induce ischemia/reperfusion over a defined time; or a permanent occlusion of the artery by fixing the ligature. The success of occlusion can be evaluated with electrocardiogram (ECG) and the macroscopic changes in the left ventricle (LV) as well as its paling.

Another important step prior to the surgery is intubation. While in most cases, intubation is performed via tracheotomy or via oral insertion of the tube under vision by a skin incision at the throat, this protocol describes the endotracheal intubation of the anesthetized animal which reduces breathing difficulties or infections post-operatively ${ }^{4,5}$. To avoid post-operative complications, air is removed from the thorax via a syringe before closing the chest.

The second task of this article is the evaluation of hemodynamic function via an isolated working heart experimental model, how it is used in other projects within our institute 6,7 . While echocardiography, cardiac magnetic resonance imaging (MRI) and invasive quantification of pressure-volume loops are well-known and widely used methods to assess cardiac function in vivo, they are known to have some limitations. Invasive approaches, such as the use of catheters to examine the global function or specific parameters of the heart, are commonly used and represent the gold standard of cardiac measurements. In contrast, the ex vivo working heart apparatus is rarely used because of its complexity and cost. There are many important aspects, from the mixture of the perfusate to the adequate cannulation of the heart, which are crucial for successful evaluation. The isolated working heart apparatus was firstly described by Oskar Langendorff in $1897^{8}$ and has been modified over the recent decades ${ }^{9}$. Today, there are two models used: 
the Langendorff (LD) mode and the working heart (WH) mode. In our study, the LD mode is used to acclimatize the heart to its new environment (about $15 \mathrm{~min}$ ). In this mode, the heart is cannulated via the aorta and the coronary arteries are perfused anterogradely, adequately supplying the myocardium. In the LD mode, the heart is not performing any pressure-volume work. By contrast, in the $\mathrm{WH}$ mode, the left atrium is cannulated via a pulmonary vein, through which the perfusate enters the left atrium. The heart then pumps this perfusate physiologically against a predefined afterload. By increasing the afterload over time, cardiac function can be continuously measured. Parameters such as coronary flow, cardiac output (CO), stroke volume (SV) and work, atrial flow and LV systolic and diastolic pressures can be measured. The impact of various treatments directly and solely on the heart can be investigated 6,10 . A review by Liao and Podesser ${ }^{9}$ presented the widespread use of this method in the evaluation of pharmacological effects on cardiac function and metabolism as well as in the exploration of various diseases such as MI, HF, obesity and diabetes.

In summary, this protocol presents a reproducible method to perform $\mathrm{MI}$ or myocardial ischemia/reperfusion (MIR) injury in vivo. In addition, it permits the characterization of LV (dys-)function on an isolated rat heart after MI. This protocol presents a unique combination of treatment and analysis.

\section{Protocol}

The experimental protocol which delivered the results described in this article has been approved by the regional Ethics Committee for Laboratory Animal Experiments at the Medical University of Vienna and the Austrian Federal Ministry of Education, Science and Research (BMWFW-66.009/0023-WF/V/3b/2016). All experiments conform with the Guide for the Care and Use of
Laboratory Animals, published by the US National Institutes of Health (NIH Publication No. 85-23, revised 1996).

NOTE: 10-12-week-old male Sprague Dawley rats of 250-300 g body weight (BW) are used. As the following procedures and treatments are performed in a sterile environment of an operating room (OR), wear scrubs, gloves, facemasks and hoods when handling animals. Before entering the OR, ensure that hands are washed and disinfected. If the intention is to operate on several animals in a surgical session, either wash and disinfect, or autoclave the instruments in between operations. These hygienic guidelines are valid for all procedures presented in the protocol section.

\section{Preoperative preparation and anaesthesia}

1. Initiate preoperative anaesthesia by injecting a mixture of xylazine (4 mg/kg BW) and ketamine (100 mg/kg BW) intraperitoneally.

2. Intubate the rats with a $14 \mathrm{G}$ tube and volumecontrolled ventilation with a mixture of $\mathrm{O}_{2}$, air and isoflurane (1-2.5\%) at $75-85$ strokes $/ \mathrm{min}, 100 \mathrm{~mL} /$ stroke/ BW (Figure 1A). If necessary, for a better view while intubating: apply Xylocain via a cotton-wool tip on the lower pharynx to achieve local relaxation.

3. Place the rats on a heated operating table in a supine position and fix the forelimbs with tape (Figure 1B).

4. Measure rectal temperature with a probe.

NOTE: It should be maintained between $37.5-38.5^{\circ} \mathrm{C}$.

5. Shave the thorax and clean the operating area with antiseptic povidone iodine solution. Apply eye ointment to the rat to prevent drying of the eyes.

6. Administer intraoperative analgesia by injecting piritramide $(0.1 \mathrm{~mL} / \mathrm{kg} \mathrm{BW})$ intraperitoneally. 
7. Place ECG probes subcutaneously in the extremities of the animal.

8. Check tail and toe reflexes prior to initiating the surgical procedure.

\section{Surgical procedure-induction of myocardial ischemia}

1. Perform skin incision using a scalpel. Ensure to start 2 $\mathrm{mm}$ parasternal on the left thorax at the level of the 3rd intercostal space and continue to the anterior axillary line at the level of the 5th intercostal space (Figure 1C).

2. Replace the superficial muscles gently to make the ribs visible (Figure 1D).

3. In the case of minor bleeding, use a cauter to obliterate or to disconnect the surrounding tissue.

4. Perform the thoracotomy at the level of the 4 th intercostal space and insert a retractor to gain visibility of the heart and the lung (Figure 1E). Carefully open the pleura to avoid bleeding.

5. Temporarily occlude the LAD using a tourniquet to induce ischemia/reperfusion (MIR) over a defined time; or permanently (MI) occlude it by making 6-7 knots using a 6-0 suture to close the ligation (Figure 1F,G).

NOTE: The right spot for occlusion of the LAD is located about $2-3 \mathrm{~mm}$ beneath the left auricle on the ventral/ left lateral margin of the heart. Successful occlusion is associated with ECG changes (ST-segment elevation) and macroscopic changes in the LV as paling.

6. In the case of the ischemia/reperfusion model, reopen the LAD by removal of the tourniquet after $30 \mathrm{~min}$ of occlusion.

7. Close the thorax with three single button sutures using a 4-0 single monofilament suture (Figure $1 \mathrm{H}$ ). Prior to tightening the last suture, remove any residual air from the thorax with a $10 \mathrm{~mL}$ syringe to prevent a pneumothorax (Figure 1I).

8. Reposition the muscles and turn off the volatile anaesthesia.

9. Suture the skin with a continuous suture using a 4-0 suture (Figure 1J).

10. Administer an antiseptic spray to protect against infections and biting of the suture by rats.

\section{Postoperative treatment and exclusion criteria}

1. Keep the rats on the heating table until they wake up. Extubate the rats as soon as they commence breathing spontaneously.

2. Put the extubated rats in a cage under a heating lamp to prevent them from cooling

3. Return rats to the animal houses under standardized conditions when they commence behaving normally again.

4. Add 2 ampules of piritramide and $30 \mathrm{~mL}$ of $5 \%$ glucose to $250 \mathrm{~mL}$ of water for post-operative analgesia for three days.

5. Check the fitness and behavior of rats with the checklist and exclusion criteria (Table 1). Observe the animals twice a day for the following week, then twice a week.

NOTE: In accordance with international standards, present any suffering animals, or animals that gain up to 6 points in the evaluation with the checklist, to veterinarians to make therapy-related decisions. Any animals that gain 7 or more points must be immediately sacrificed with an overdose of ketamine and xylazine. 


\begin{tabular}{|c|c|c|}
\hline Examination & Observation & Score \\
\hline \multirow[t]{5}{*}{ Body weight } & stable & 0 \\
\hline & $10 \%$ loss & 4 \\
\hline & $15 \%$ loss for $48 \mathrm{~h}$ & 7 \\
\hline & $18 \%$ loss & 7 \\
\hline & normal (coat flat and shiny) & 0 \\
\hline \multirow[t]{8}{*}{ External appearance } & piloerection & 1 \\
\hline & haematoma & 2 \\
\hline & skin wounds/cuts/bite marks & 2 \\
\hline & severely reduced grooming & 4 \\
\hline & (orifices unclean/clotted or moist) & 7 \\
\hline & severe skin irritations or wounds & 7 \\
\hline & hunched posture $>2 \mathrm{~h}$ & 7 \\
\hline & $\begin{array}{l}\text { significant abdominal } \\
\text { distension (ascites) }\end{array}$ & 7 \\
\hline \multirow[t]{9}{*}{ Behavior } & $\begin{array}{l}\text { normal (sleeping, curious, social } \\
\text { contacts, reaction when touched) }\end{array}$ & 0 \\
\hline & unusual behaviour, e.g. impaired activity & 2 \\
\hline & $\begin{array}{l}\text { self-isolation, pronounced } \\
\text { hyperactivity or stereotypia }\end{array}$ & 4 \\
\hline & lethargia for $<6 \mathrm{~h}$ & 4 \\
\hline & lethargia for $6 \mathrm{~h}$ to $8 \mathrm{~h}$ & 7 \\
\hline & apathia $>8 \mathrm{~h}$ & 7 \\
\hline & $\begin{array}{l}\text { stereotypia uninterrupted for }>10 \\
\text { min. and still persisting after } 2 \mathrm{~h}\end{array}$ & 7 \\
\hline & signs of pain when touched & 7 \\
\hline & automutilation & 7 \\
\hline Digestion & normal & 0 \\
\hline
\end{tabular}




\begin{tabular}{|l|c|c|}
\cline { 2 - 3 } & diarrhea (soft feces) & 3 \\
\cline { 2 - 3 } & diarrhea for 72 h or watery & 7 \\
\cline { 2 - 3 } & bloody stool & 7 \\
\hline
\end{tabular}

Table 1: Checklist and exclusion criteria. This table contains the examinations that must be observed and the corresponding score. Accordingly, the post-operative treatment of the animal must be adapted, or a veterinarian must be consulted.

\section{Echocardiography measurements}

NOTE: Echocardiography is usually performed twice, prior to the induction of $\mathrm{MI}$ and before the organs are harvested.

1. Inject rats with a mixture of xylazine $(4 \mathrm{mg} / \mathrm{kg} \mathrm{BW})$ and ketamine (100 mg/kg BW) intraperitoneally.

2. Place the rats in a supine position on a heating tray. Apply echo gel to the chest, which helps the ultrasound waves travel better and reduces signal interferences.

3. Obtain parasternal short axis views of the LV cavity at the level of the papillary muscle.

4. Perform M-mode echocardiography in order to measure left ventricular ejection fraction and morphology.

\section{Organ harvesting (without working heart)}

1. Administer xylazine (4 mg/kg BW) and ketamine (100 mg/ $\mathrm{kg} \mathrm{BW}$ ) intraperitoneally prior to organ harvesting. Ensure that the reflexes are negative.

NOTE: No intubation is required as the procedure does not last longer than $1 \mathrm{~min}$.
2. Use a scalpel to make a skin incision under the xiphoid and extend it parallelly to the ribs on both sides using scissors.

3. Cut the ribs in the frontal axillary line and grab the xiphoid to lift the chest up (Figure 2A).

4. Remove anatomical or fibrotic tissue adhesions by carefully rupturing the tissue with two pairs of forceps.

5. Take blood samples (for blood gas evaluation or molecular analyses) from the vena cava inferior with a 5 $\mathrm{mL}$ syringe.

6. Perform the excision of the whole heart at the inlet and outlet level (Figure 2B). If necessary, proceed with working heart evaluation as described in section 6.

7. Harvest organs, shock frost them in liquid nitrogen and store in $-80{ }^{\circ} \mathrm{C}$ for further molecular analyses, or in formaldehyde for histological purposes.

\section{Ex vivo hemodynamic measurements via a working heart system}

NOTE: The general setup and the components of the apparatus has been previously described ${ }^{11}$. The following protocol describes the handling of the animal's heart and the necessary steps to evaluate LV function. 
1. Anesthetize rats as described in step 5.1 and inject 200 IU of heparin intravenously (femoral vein).

2. Open the thorax via an incision beneath the costal arch with a scalpel and extend it to both anterior axillary lines with scissors and elevate the sternum.

3. Cut the great vessels near their outlet or inlet to the heart to excise it (Figure 2B).

4. Immerse the heart in ice-cold Krebs-Henseleit buffer and mount it on the erythrocyte-perfused isolated heart system via cannulating the aorta (Figure 3A).

5. Start with the LD mode with a constant afterload of 60 $\mathrm{mmHg}$ (stabilization period).

6. After 15 min of LD mode, switch to the $\mathrm{WH}$ mode. Therefore, cannulate the left atrium via a pulmonary vein (Figure 3B). Then, change the flow direction in the system by opening the clip that occludes the atrial cannula. This results in a perfusion of the left atrium and a physiological blood flow in the left heart ${ }^{11}$.

7. Record hemodynamic measurements for $20 \mathrm{~min}$ in the WH mode.

8. Collect blood drops of the coronaries with a $2 \mathrm{~mL}$ syringe to measure coronary flow (CF, $\mathrm{mL} / \mathrm{min}$ ) every $5 \mathrm{~min}$.

NOTE: CF is measured as the difference between left atrial flow (LAF) and aortic flow (AF).

9. Perform continuous measurements of LAF (equivalent to cardiac output) and AF with a flow probe.

NOTE: The probe is inserted via the $\mathrm{WH}$ apparatus into the LV. All data are continuously registered.

10. If the ongoing protocol requests, insert a high-fidelity catheter retrogradely via the aortic valve into the LV and measure the left ventricle systolic pressure (LVSP).
11. To assess the pressure-volume work performed per minute, calculate stroke volume as cardiac output divided by heart rate.

12. Calculate external heart work (EHW) according to the following formula: CO $\times$ LVSP $(g \times \mathrm{m} / \mathrm{min})$ normalized to heart weight.

\section{Representative Results}

The following results have been published by Pilz et al. ${ }^{6}$. With this precise surgical procedure, the cardioprotective effect of remote ischemic perconditioning (RIPerc) can be investigated. This is a potential new treatment for patients suffering from acute MI or MIR and subsequent ventricular remodeling, which in many cases leads to consecutive HF. Mimicking the pathophysiological changes of MI/MIR is an obligatory step in the evaluation of treatments as in vitro or ex vivo studies do not provide the physiological environment. In this protocol, the animals were subjected to 30 min of LAD occlusion followed by reperfusion (i.e., MIR).

To prove the reproducibility of the procedure, histological cuts and stains were performed (Figure 4A). It was clear that the fibrotic scar in MIR+RIPerc treated animals was comparable with the scar formation of the Sham animals while the comparison of fibrosis between Sham and MIR groups was significant (Figure 4B). Additionally, MIR+RIPerc treated animals showed significantly reduced fibrosis compared to MIR-treated animals. However, the representative histological images clarify the potency of this surgical procedure as the infarction is explicitly sustained in the MIR group (Figure 4A). Using in vivo echography, ejection fraction, LV end-diastolic and endsystolic diameters (LVEDD and LVESD) were measured and showed significantly reduced cardiac function due to MIR 
treatment while hemodynamic parameters were preserved by

RIPerc (Figure 4C-F). Ex vivo hemodynamic data exhibited the effectiveness of the procedure as the MIR group showed significant decreases in LVSP, cardiac output (CO), stroke volume (SV) as well as external heart work (EHW) (Figure 5A-G).
A literature search about this surgical procedure reported no negative or unsatisfying comments and results when it was adequately performed. Nevertheless, pitfalls mentioned in the introduction and the discussion need to be prevented and training is mandatory to acquire a stable level of performance and to obtain comparable results. 


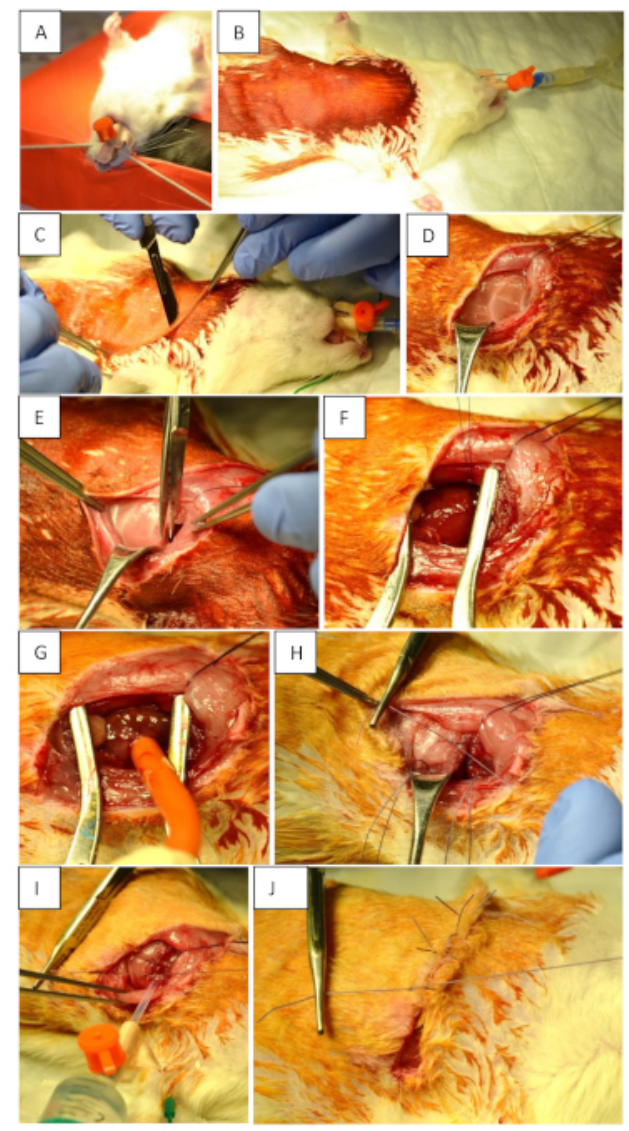

Figure 1: Preoperative preparation and surgical procedure. (A) Intubation of the animal using a $14 \mathrm{G}$ tube. (B) Supine positioning and disinfection of the surgical field. (C) Skin incision (2 $\mathrm{mm}$ parasternal on the left thorax at the level of the 3rd intercostal space). The incision must reach the anterior axillary line at the level of the 5th intercostal space. (D) Displace the muscles to make the ribs visible. (E) Opening of the thorax. (F) Permanent occlusion of LAD using 6-7 knots. (G) Transient occlusion of LAD using a tourniquet. $(\mathbf{H})$ Closure of the chest after myocardial ischemia and reperfusion by placing three single-knot sutures around the ribs. (I) Proper closing of the thorax. Use a $10 \mathrm{~mL}$ syringe to remove any residual air from the thorax before fixing the last knot tightly. This is integral to prevent a pneumothorax. $(\mathbf{J})$ Skin suture. Please click here to view a larger version of this figure. 


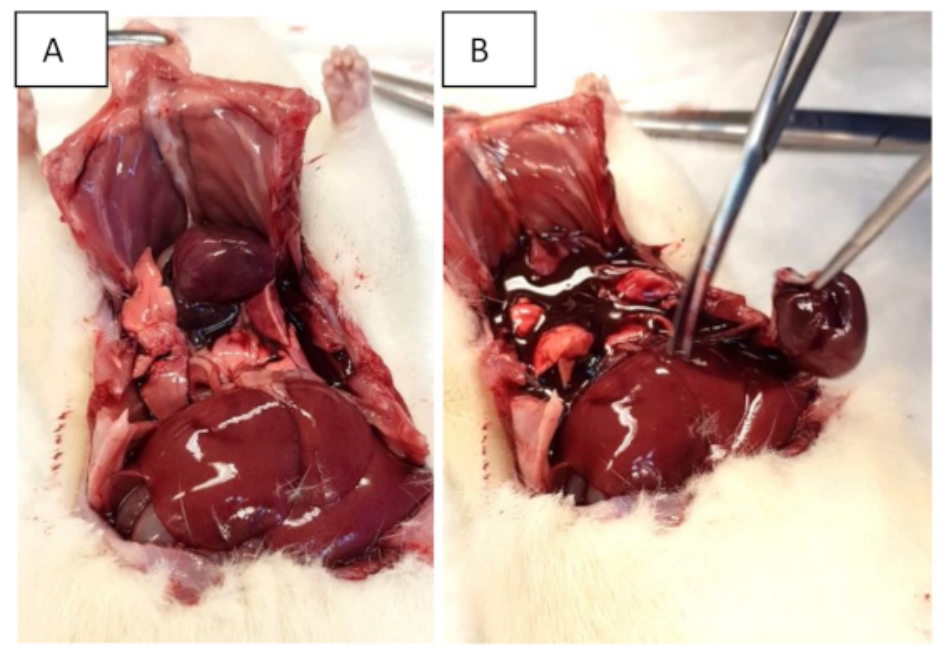

Figure 2: Organ harvesting. (A) Open the chest with sub-xiphoidal cuts and extend them to both mid-axillary lines. Further cuts through the ribs are performed to facilitate lifting of the sternum. (B) Excision of the heart. Please click here to view a larger version of this figure. 


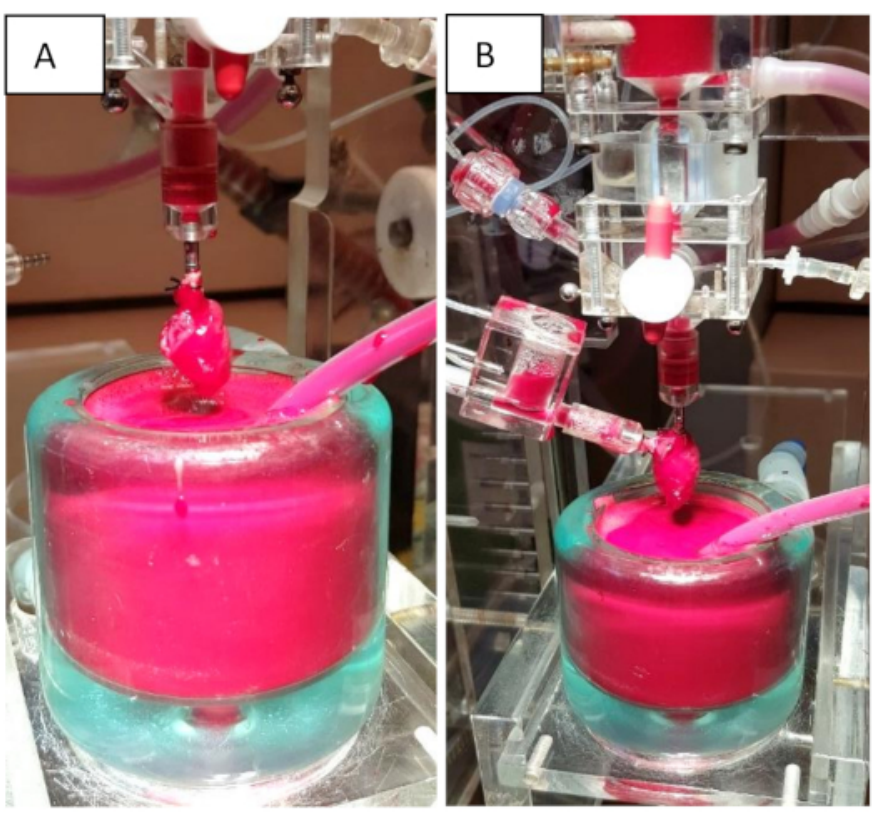

Figure 3: Isolated heart apparatus. (A) Langendorff mode. The heart is mounted to the WH apparatus via cannulation of the aorta. (B) Working heart mode. The system can be switched to the WH model to evaluate cardiac function by cannulating the left atrium. Please click here to view a larger version of this figure. 
A

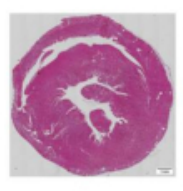

Sham

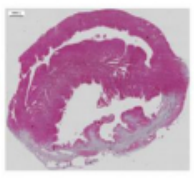

MIR

C
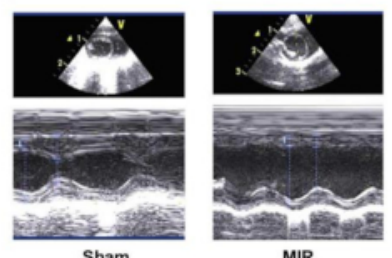

MIR

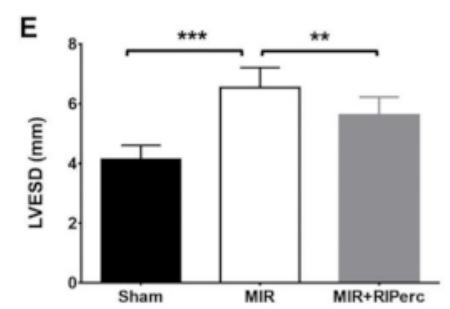

B

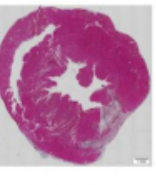

MIR+RIPerc

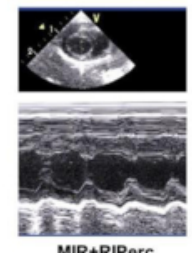

MIR+RIPerc

D
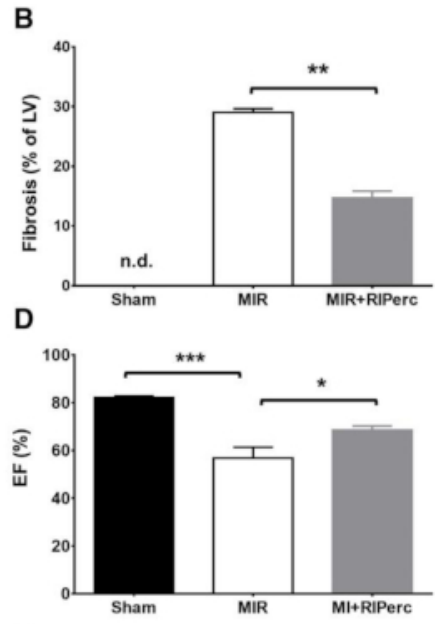

F

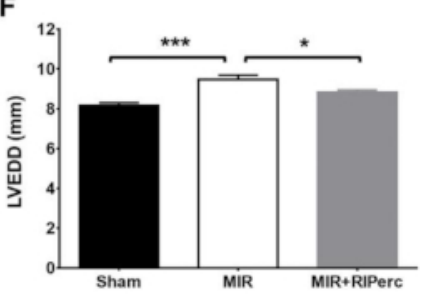

Figure 4: Effect of remote ischemic conditioning on scar formation, left ventricular function and remodeling. (A)

Histological LV slices harvested on day 14 post-myocardial reperfusion. (B) Quantified results of fibrosis in bar graphs. (C) Representative M-mode echocardiograms. (D) Ejection fraction (EF) quantified in bar graphs. (E) LV end-systolic diameter (LVESD) quantified in bar graphs. (F) LV end-diastolic diameter (LVEDD) quantified in bar graphs. MIR, myocardial ischemia-reperfusion; RIPerc, remote ischemic perconditioning. Data are expressed as mean \pm SEM. ${ }^{*} p<0.05 ;{ }^{* *} p<0.01$; ${ }^{* * *} p<0.001$. Reprinted from Pilz et al. ${ }^{6}$ with permission from Elsevier. Please click here to view a larger version of this figure. 

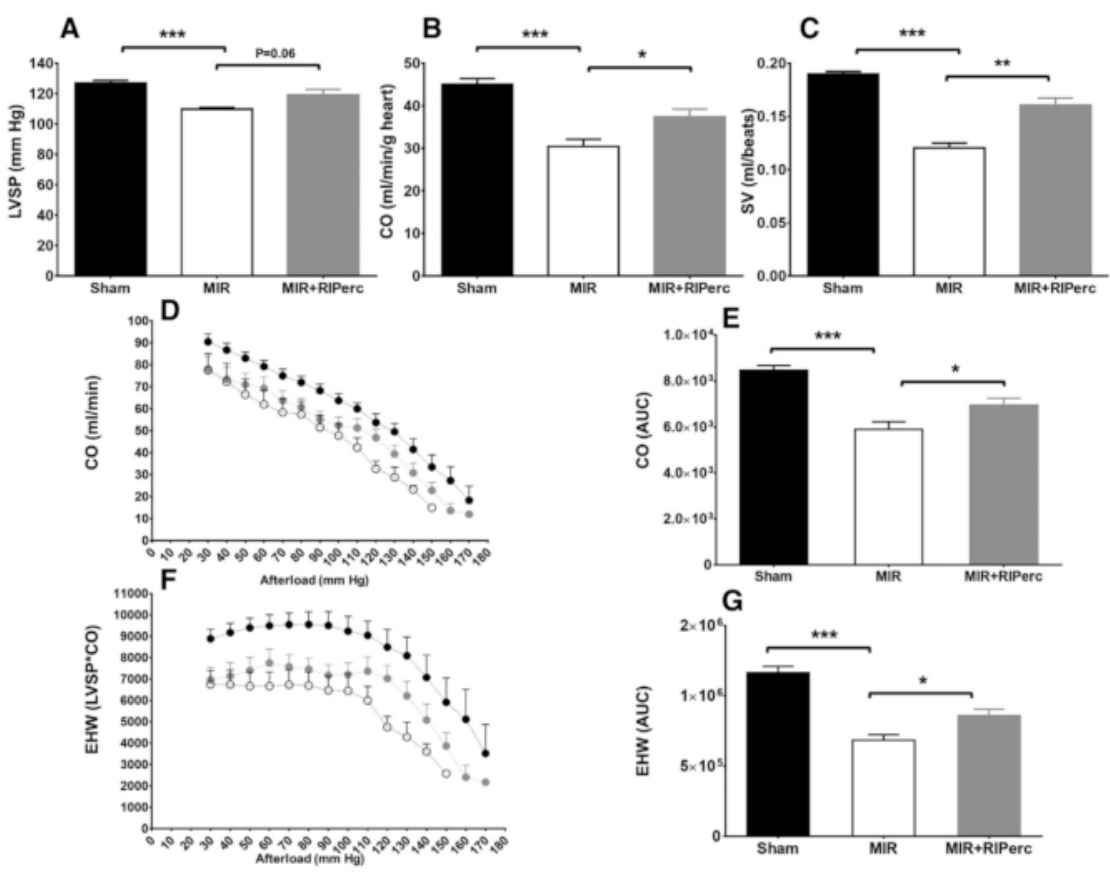

G

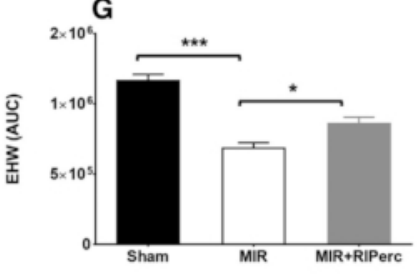

Figure 5: Effect of RIPerc on LV hemodynamic function. (A) LV systolic pressure (LVSP), (B) cardiac output (CO), and (C) stroke volume (SV) results were obtained from the isolated working heart on day 14 post-myocardial reperfusion. (D) CO is depicted as a function of afterload; $(\mathbf{F})$ external heart-work as function of afterload, quantified results in bar graph ( $\mathbf{E}$ and G). Data is expressed as mean \pm SEM and $n=4-7$ per group. ${ }^{*} p<0.05$; ${ }^{* *} p<0.01$; ${ }^{* \star} p<0.001$. MIR, myocardial ischemia/ reperfusion; RIPerc, remote ischemic preconditioning; EHW, external heart work; SV, stroke volume; AUC, area under the curve. Reprinted from Pilz et al. ${ }^{6}$ with permission from Elsevier. Please click here to view a larger version of this figure.

\section{Discussion}

Adverse remodeling post-MI is considered to be a key mechanism in the development of heart failure. Therefore, to ensure the continuity of cardiovascular research, experimental procedures and techniques should be reproducible. A comprehensible and clearly defined experimental protocol is a fundamental element of reproducibility. Reproducibility refers to results that can be repeated by multiple scientists and are validated across laboratories. This study aimed to present a semi-minimally invasive method to induce chronic or re-perfused $\mathrm{Ml}$ and assess the cardiac hemodynamic function in rats.

These results and further published data show the high potency of this surgical method and its importance in research on $\mathrm{MI}$, remodeling and $\mathrm{HF}$. While ischemia/reperfusion injury can be used to understand the changes in $\mathrm{Ml}$ with subsequent reperfusion, permanent occlusion allows further understanding of the short- and long-term remodeling processes of the myocardium. Other surgical approaches cause more tissue damage and animals show higher risks of developing infections and pneumothorax, resulting in higher 
drop-out rates. In contrast, this procedure is aimed to reduce mortality by specific improvements in the setup and handling. Additionally, they show variations in fibrotic scar expansion due to unstable LAD occlusion.

Our protocol provides an easy method for intubation, which is one of the most critical steps of the whole procedure. In contrast to several other publications ${ }^{12}$, tracheotomy is not performed in our procedure. This enhances awakening and rehabilitation of the animals post-operatively, leading to the development of the pathophysiological changes which are intended by this surgical procedure before the animals undergo post-operative measurements. Obviously, if it is a non-survival protocol, tracheotomy is performed under vision and is thus easier to perform. Additionally, closing of the tracheotomy in a survival-protocol is not applicable. If the thorax is opened, it is mandatory to ventilate the lung to prevent collapse. Therefore, the rats are intubated prior to the surgical procedure. The minimally invasive approach does not cut the ribs or sternum thus maintaining the compactness and stability of the thorax. Consequently, the animals' recovery is improved, and the risk of spontaneous pneumothorax or bleeding is relatively low.

As aforementioned, while the intubation is of clear advantage, it is difficult to perform and may cause a higher drop-out rate at the beginning of the experiments. This problem can be mitigated with training and some anatomical knowledge. It is important to insert the tube at the right angle and stretch the animal's body until the light shines through the vocal lips after which the tube can be gently pushed forward. Take care to not harm the vocal lips as this can cause swelling, subsequent occlusion of the glottis and suffocation.

It is also important that the LAD is ligated correctly. The small surgical window, fast beating heart, and ventilated lung (avoid touching it as much as possible as every contact may result in bleeding in the lung) render the vessel not clearly visible. Therefore, anatomical knowledge is indispensable. The left auricle is indispensable to help standardize both the area at risk and to position the ligation around the LAD. The stitch needs to be performed intramurally, not transmurally in the LV as this may cause a reduction of the LV chamber diameter and volume which is not due to the pathological processes. Successful occlusion is associated with cyanosis of the myocardial area at risk and elevation of ST-segment on ECG. The main limitation of this procedure is the correct positioning of the suture. To achieve comparable results, the stitches must be at the same level and need to use similar amounts of tissue. This requires a high level of training and the different weights of the animals must be considered. Another point to consider is the adequate removal of the pneumothorax prior to closure of the intercostal space. If this is not precisely performed, the animals will exhibit difficulties in breathing as inflation of the left lung will be hindered by a pneumothorax. As aforementioned, this can be mitigated by using a syringe to remove any residual air from the thorax.

Currently, this MI procedure is a commonly used method which guarantees comparable results and a high survival rate if the critical steps are performed with high precision. Future projects on various treatments, devices or drugs in MI, HF or cardiac remodeling can be evaluated by performing this minimally invasive technique.

The WH measurements are, as aforementioned, not commonly used as its maintenance and handling requires specific equipment and knowledge. To acquire representative and comparable data, pitfalls must be avoided. The most critical steps are the mounting of the heart and switching from the $\mathrm{D}$ model to the $\mathrm{WH}$ mode. If the heart is not excised 
adequately, the mounting may be difficult as sufficient aortic tissue length is required to fix the heart to the apparatus. Soon after connecting to the LD mode, the heart frequency may decrease due to the washing in cold buffer, the disconnection of its physiological stimuli in the body or the reperfusion with blood from another species by the apparatus. In such cases, a pacemaker must be applied to both restore and preserve the physiological frequency. This ensures comparable results in all animals. As the blood volume within the apparatus is a multiple of the physiological volume in rats, bovine red blood cells in a Krebs-Henseleit buffer-based suspension are used.

The switch from the LD mode to the WH mode is synonymous to a switch from passive to active heart work. The LD mode is used to accustom the heart to its new environment. In the WH mode, the heart must perform its physiological ejection functions. Therefore, a short adaption phase to the new circumstances is required before the evaluation by increasing the afterload.

Another critical step which is commonly forgotten is the adequate preparation and maintenance of the apparatus and the perfusate. The precise volume of each compound must be mixed and the temperature within the system must be controlled and adjusted. Nevertheless, the WH is an elegant method to assess cardiac output, stroke volume, left ventricular systolic pressure and coronary flow simultaneously.

This highly reproducible procedure to induce $\mathrm{Ml}$ and the representing data acquired by the $\mathrm{WH}$ apparatus are proving their capability themselves. The semi-minimally invasive approach, the level of LAD occlusion and intubation method facilitate fast recovery and low variability in infarct size.
Additionally, cardiac function analysis in isolated working hearts provide valuable hemodynamic results.

\section{Disclosures}

The authors have nothing to disclose.

\section{Acknowledgments}

The authors thank the operation theater team and technicians of the Center for Biomedical Research for their contribution, technical assistance, valuable input and advice. The projects are funded by the Ludwig Boltzmann Institute, Cluster for Cardiovascular Research (REM project).

\section{References}

1. WHO. Global Health Estimates 2015: Deaths by Cause, Age, Sex, by Country and by Region, 2000-2015. World Health Organization (2016).

2. Jaquet, K. et al. Reduction of myocardial scar size after implantation of mesenchymal stem cells in rats: what is the mechanism? Stem Cells and Development. 14 (3), 299-309 (2005).

3. Liu, P., Xu, B., Cavalieri, T. A., Hock, C. E. Agerelated difference in myocardial function and inflammation in a rat model of myocardial ischemia-reperfusion. Cardiovascular Research. 56 (3), 443-453 (2002).

4. Kolk, M. V. V. et al. LAD-Ligation: A Murine Model of Myocardial Infarction. Journal of Visualized Experiments. (32), e1438 (2009).

5. Lugrin, J., Parapanov, R., Krueger, T., Liaudet, L. Murine Myocardial Infarction Model using Permanent Ligation of Left Anterior Descending Coronary Artery. Journal of Visualized Experiments. (150), e59591 (2019). 
6. Pilz, P. M. et al. Remote ischemic perconditioning attenuates adverse cardiac remodeling and preserves left ventricular function in a rat model of reperfused myocardial infarction. International Journal of Cardiology. 285, 72-79 (2019).

7. Santer, D. et al. In vivo and ex vivo functional characterization of left ventricular remodelling after myocardial infarction in mice. ESC Heart Failure. 2 (3), 171-177 (2015).

8. Langendorff, $\mathrm{O}$. Untersuchungen am überlebenden Säugetierherzen II. Über den Einfluss von Wärme und Kälte auf das Herz der warmblütigen Tiere. Pflügers Archiv für die gesamte Physiologie des Menschen und der Tiere. 66 (67-68), 355-400 (1897).

9. Liao, R., Podesser, B. K., Lim, C. C. The continuing evolution of the Langendorff and ejecting murine heart: new advances in cardiac phenotyping. American Journal of Physiology-Heart and Circulatory Physiology. 303 (2), H156-167 (2012).

10. Podesser, B. K. et al. The erythrocyte-perfused "working heart" model: hemodynamic and metabolic performance in comparison to crystalloid perfused hearts. Journal of Pharmacological and Toxicological Methods. 41 (1), 9-15 (1999).

11. Kiss, A. et al. Argon preconditioning enhances postischaemic cardiac functional recovery following cardioplegic arrest and global cold ischaemia. European Journal of Cardio-Thoracic Surgery. 54 (3), 539-546 (2018).

12. Kiss, A. et al. Vagal nerve stimulation reduces infarct size via a mechanism involving the alpha-7 nicotinic acetylcholine receptor and downregulation of cardiac and vascular arginase. Acta Physiologica. 221 (3), 174-181 (2017). 